\title{
Las Descripciones Geográficas de Indias y un Primer Diccionario de Americanismos
}

POI MIGUEL A. UGARTE CHAMORRO.

Es fácil suponer la sensación de estupor que produjo en Europa, y principalmente en España, el regreso de Cristóbal Co. lón con la increíble noticia del descubrimiento de nuevas tierras en su epopéica aventura, en pos de nuevas rutas a las codiciadas Indias Occidentales. Explicable es, también, cómo ese interés, acompañado de gran curiosidad, iba en aumento a medida que se iban conociendo mayores informaciones, todas ellas a cual más fantásticas, con el propósito de dar idea de un mundo totalmente diferente al conocido hasta entonces. Portugal y España fueron los primeros reinos que comprobaron, en cierta forma, la veracidad de ese nutevo mundo al admirar de cerca la extrañeza de sus gentes, la variedad de sus exóticos frutos y las muestras de los metaléblipteciosos @yrarras especies no menos codiciadas, cuando el genovés presentero las irrecusables pruebas de la gran aventura.

Imposible sería, más bien, describir, por otro lado, el choque impresionante experimentado por los descubridores en su legendaria hazaña. La contemplación de paisajes nunca vistos y continuamente renovados; la objetiva desproporción americana percibida por la sensibilidad de estos primeros hombres que amasaron su lenguaje interior con sólo la experiencia de su sencillo y tibio pueblo, o a lo más, con el entorno regional de su país que transitaron sin angustia; las multiformes especies de animales y la matizada variedad de un paradisíaco mundo vegetal; todo ello, movió el ánimo de los descubridores y, posteriormente, el de los conquistadores y colonizadores para describir y relatar las nuevas experiencias.

Desde España, la corona española y los hombres de empresa -capitanes, soldados, religiosos, comerciantes y aventureros- exigían y esperaban ansiosos, día tras día, más informaciones para trocar sus espectativas en segura decisión: llegar a las Indias en una u otra forma. 
Las primeras descripciones llevadas a España por Colón, fueron sin duda, los primeros documentos que iniciaron otra ditar el entronque y la interpenetración, tanto espiritual como suerte de riqueza, que con el tiempo, habria de sustentar y acrematerial, de los comunes intereses, valores y destinos culturales latinoamericanos.

Antes de cumplirse el año del descubrimiento de América, los Reyes Católicos, desde Barcelona, interesados personalmente en las descripciones escritas por Colón, le solicitaban mayores datos sobre la ubicación de las islas y tierras descubiertas. así como sus nombres; y al año siguiente, en 1594, reiteraban su deseo de conocer los nombres de cada lugar hallado, y de igual manera, los nombres con que las llamaban los indios.

Cuántas descripciones espontáneas se harian y cuántas noticias se proporcionarian en aquellos años en que se ensanchaba el mundo y se multiplicaban las ambiciones de todo género.

El licenciado Alonso de Zuazo, con estudios de cosmografía y geografía en Salamanca, escribía a su monarca en 1518, desde Santo Domingo, dándole segurúdades de estar en la me. jor tierra del mundo, dondéno habiendo frío ni calor, los árboles nunca perdian su follaje; tierra llena de fuentes y de ríos cargados de oro fino, y enildondeabüadaban las maderas maravillosas como el brasil, elogulayucidinoyvotras maderas medicinales, sin faltar las que sabían a canela...

Las noticias de América se sucedían a través de cartas, descripciones y relatos, pero no bastaban para satisfacer la curiosidad general y los propósitos de la Corte y las esferas oficiales. Se hizo, pues, necesario, que la antigua crónica castellana se trasladara a América por providencia real. La Corona española, interesada en gobernar las nuevas tierras que se iban sujetando a su jurisdicción, requería datos fidedignos para asegurar su imperio. En efecto, dictó las órdenes apropiadas para que los descubridores y conquistadores fueran acompañados de veedores que hicieran la descripción de esos territorios, así como de las costumbres y usos de sus moradores.

Las recomendaciones dadas a Colón para que informara sobre los descubrimientos se fueron repitiendo con otros capitanes y aventureros a quienes se exigía la descripción de los países descubiertos, conquistados o poblados, más la información sobre las gentes de esos lugares, así como de las cosas más notables que se vieran. Esta clase de ordenanzas ha formado va- 
liosa documentación conocida por los historiadores con los nombres de instrucciones y capitulaciones, que abarcan un período entre 1501 y 1573, año en que se dieron las muy completas "Ordenanzas de poblaciones y descubrimientos".

El Adelantado del Yucatán, Diego Velásquez, al enviar a Hernán Cortés, en 1518, a proseguir sus descubrimientos, le recomendaba "inquirir y saber el secreto de las dichas islas y tierras y de las más a ellas comarcanas... así de la manera y conversación de la gente de cada una de ellas en particular, como de los árboles y frutas, yerbas, aves, animalías, oro, piedras preciosas, perlas y otros metales, especería y otras cualesquier cosas que de las dichas islas y tierras pudiereis saber, y de todo traer entera relación por ante escribano..." (1)

Las descripciones y relaciones cobraron mayor importancia con motivo de las disposiciones metropolitanas acerca de la instauración del régimen de los "repartimientos" y "encomiendas", en las tierras conquistadas, con el fin de poblarlas y de designar los tributos que los indios debían rendir a la Corona y a los españoles propietarios de-esos repartimientos. El erudito americanista Jiménez de la Espada, supone que las disposiciones sobre los repartimientos y encomiendas son los antecedentes directos de las Instrucciones y Memorias que se dictaron durante el reinado deibedipea $\mathrm{IE}$ Iparas las Relaciones históricogeográficas de los domînîôs tahlitonindianos como peninsulares.

Como la lengua de los conquistadores fue el mejor vehículo de la gran aculturación en América, el castellano, que iba moldeando con sus formas las exigencias vitales y las necesidades culturales, tenía que recurrir muchas veces a los préstamos lingüísticos, así es como, al cumplirse las disposiciones oficiales, las crónicas y descripciones, desde sus comienzos recogieron las voces indígenas imprescindibles referentes a la toponimia, antroponimia, zoonimia y fitonimia, más las correspondientes a la cultura general americana, expresivas de instituciones, creencias, usos y costumbres. "El Vocabulario de Nebrija, 1493.. nos brinda la estupenda novedad de traer ya el más temprano americanismo, canoa". (2)

El castellano "no podía bastar a las singulares y múltiples necesidades de la nueva vida, y el trato con los naturales los

(1) M. Jiménez de la Espada. Relaciones geográficas de Indias, T.I., Madrid, 1881, pág.

(2) Amado Alonso. La base linguiística del español americano, en Estudios lingüísticos.

(Temas Hispano-americanos), Gredos, Madrid, 1953, pág. 28. 
hizo aprender y apropiarse muchas voces indigenas, de las cuales, unas han venido a ser universalmente conocidas y usadas, al paso que otras no se oyen ni entienden sino en ciertas comarcas..."(1).

Grave preocupación hubo de embargar al Estado español las proporciones que iban adquiriendo los descubrimientos a comienzos del siglo XVI, a los que debia atender con prontas medidas politicas y administrativas, aparte de la preocupación religiosa, hasta cierto punto indesligable de los intereses del gobierno. Por eso se creó en Sevilla, en 1503, la Casa de Contratación encargada del gobierno político de las provincias indianas. Posteriormente, en 1524, se instaló el Real y Supremo Consejo de las Indias bajo la presidencia de Fray Garcia de Loaiza, más tarde arzobispo de Sevilla. Este Consejo fue el que dirigió las actividades de los Oficiales Reales, Gobernadores, Virreyes y Audiencias que ejercieron su autoridad en América.

El Consejo de Indias, si bien es cierto, no tuvo entre sus funciones las de carácter científico, no por eso se desatendió de ellas cuando tuvieron relacion con-su tarea administrativa, como las referentes a la geografía, la Jistoria y las ciencias naturales. Esta clase de actividades, con el tiempo y el avance de la cultura, llegarian a tener insospechado valor documental para otras ciencias como la bipgitistiga yeta filología.

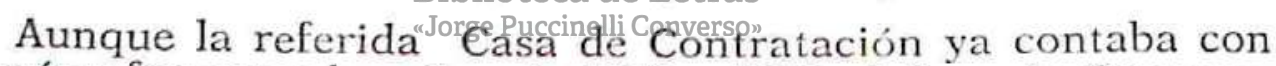
cosmógrafos entre los años de 1536 a 1556, el Consejo Supremo de las Indias creó en 1571 el cargo de "Cosmógrafo-Cronista Mayor de las Indias para investigar y escribir la historia de las colonias y para investigar y recopilar todos los asuntos de geografía, física y náutica". (2)

Felipe II, interesado en el estudio de las ciencias, prestó el apoyo del Consejo a Gonzalo Fernández de Oviedo, para la investigación de la geografía e historia de las Indias, haciendo posible la publicación de su obra en 1533. De igual manera, envió en 1570 a Nueva España y al Perú a Francisco Hernández para investigar la fauna y la flora.

Las sucesivas y variadas disposiciones oficiales dictadas desde España con el nombre de capitulaciones, inștrucciones, memorias, ordenanzas, cédulas, etc., para el mayor conocimiento y mejor administración de los territorios americanos tuvie-

(1) Rufino J. Cuervo. El castellano en América, El Ateneo. Buenos Aires, 1947, pág. 79.

(2) Ernesto Schafer. El Consejo Real y Supremo de las Indias, T.I., Sevilla, 1935, pág. 118. 
ron que perfeccionarse por la experiencia progresiva y por los objetivos políticos cada vez más precisos. Respondiendo a tales mandatos y recomendaciones se sucedió importante y nutrida información entre la cual nos interesa destacar las "Relaciones Geográficas" y que correspondieron a diferentes interrogatorios que formularon las autoridades españolas.

La inclinación del monarca Felipe II por las ciencias, como acabamos de mencionar, y su preocupación por el buen gobierno de ultramar fueron secundados decididamente por don Juan de Ovando, diligente y culto funcionario del Consejo General de la Inquisición que en 1569 fue nombrado Visitador del Consejo de Indias y, posteriormente, su Presidente, en 1571. Uno de los primeros actos de Ovando, fue el de recopilar las leyes dictadas para la administración de las Indias. En esta forma se publicaron las Ordenanzas Reales del Consejo, en las cuales se establecía la elaboración de un libro que contuviera todas las descripciones hechas sobre las provincias americanas, el que debía ser escrito por el cosmógrafo y cronista. Así, por vía de ejemplo, la ordenanza 120 disponía: ...."el Cosmógrafo recopile y vaya siempre coligiendo la historia natural de las yerbas, plantas, animales, aves, pescados yotras cosas dignas de saberse, que en las provincias, islas, mares y ríos de las Indias hubiere, según que lo pudiere hacer por las descripciones y avisos que se enviaren de aquellastpartes"

Por la misma época, gracias al talento organizador de Ovando se inició en el Consejo la preparación de detallados interrogatorios con el fin de llevar una estadística sobre puntos concernientes a la historia, la geografía y las ciencias naturales, importantísima medida para esos lejanos días, que no tiene que envidiar a las modernas encuestas, y que dio lugar a la redacción de las Relaciones histórico-geográficas. El fruto de esos interrogatorios no dejó de esperarse ya que el cosmógrafo-cronista Juan López de Velazco las aprovechó para publicar en 1574 su magnífica "Descripción Universal de las Indias".

Los interrogatorios que originaron las Relaciones Geográficas, (1569), estuvieron constiturdos, en un principio, por $37 \mathrm{ca}$ pítulos. En 1571 hubo interrogatorios con 200 , y en 1575 , se redujeron a 135 .

Más tarde, López de Velazco continuó la labor de su maestro Ovando, disponiendo que las Relaciones se hicieran no ya en

(1) Jiménez de la Espada. Obra citada T. I. pág. LXII. 
el Consejo sino en la sede del pueblo, donde se hacia la encuesta y por las autoridades correspondientes, para lo cual se enviaba la instrucción y memoria a fin de realizarlas adecuadamente.

El historiador Raúl Porras Barrencchca, al referirse a las Relaciones Geográficas, manifiesta que "tienen un duble caracter: histórico y geográfico. Describen la,tierra, el temple o la fertilidad de ésta, las aguas, los vientos, los frutos, los a wimales, las canteras, los pastos y otras caracteristicas naturales; pero al mismo tiempo se ocupan del nombre antiguo del pueblo, de su etimología, de cómo gobernaban los antiguos señores, sus guerras, adoraciones, trajes y "costumbres buenas y malas que te nían". También averiguan quién fundó el pueblo de españoles y las transformaciones sufridas por éste". (1)

Las Relaciones Geográficas que se ocupan del Perú traen la descripción más detallada de sus más importantes provincias y ciudades en el siglo XVI. Respondieron a un cuestionario impreso, compuesto por 355 capítulos, hecho en el año 1604 por el Consejo de Indias, cuando lo presidia don Pedro Fernández de Castro, Conde de Lemos y de Andrade, político ilustre que para nosotros cobra singular importaneja por ser el autor del primer trabajo de lexicografia amiericana, a nuestro parecer.

El referido cuestionario es un fol. de $88 \mathrm{ff}$. que lleva por título: "Interrogatorio para todas las ciudades, villas y lugares de españoles y pueblos naturahtess dists,s Indias Occidentales, islas y Tierra Firme; al cual se ha de satisfacer conforme a las preguntas siguientes, habiéndolas averiguado en cada pucblo con puntualidad y cuidado". (2)

Estas Relaciones comenzaron a publicarse por Marcos $\mathrm{Ji}$ ménez de la Espada, en Madrid, con motivo de la celebración del Congreso Internacional de Americanistas de 1881.

Publicados tan interesantes documentos sobre la historia y la geografía del Perú, constituyen una valiosa fuente, muy conocida por los estudiosos, mas entendemos que no se les ha dado la debida importancia como fuente documental linguística y filológica. Este es nuestro propósito: presentarlos como marco adecuado para relievar un significativo dato, inserto justamente en estas Relaciones: el primer Diccionario de Americanismos, importante no tanto por el acopio de voces registradas,

(1) Raúl Porras Barrenechea. Fuentes históricas peruanas, Mejía Baca-Villanueva, Lima, 1955, pág. 221.

(2) Rubén Vargas Ugarte. Manual de estudios peruanistas, Studium, Lima, 1952, pág. 20. 
que las trae en escaso número, sino por la época en que fue escrito, y aún más, por el título expreso de "Diccionario" que le dio el autor, referido a "los vocablos particulares de las Indias, y poco familiares en España", como dice textualmente el original.

Don Pedro Fernández de Castro Andrade y Portugal, séptimo Conde de Lemos, fue hijo de Fernando Ruiz de Castro Andrade y Portugal, que llegó a ser Virrey de Nápoles, y de doña Catalina de Zúñiga y Sandoval, emparentada cercanamente con San Francisco de Borja. Nació en Monforte de Lemos alrededor de 1576 y casó con la Condesa Catalina de la Cerda y Sandoval, prima hermana suya e hija de Francisco Gómez de Sandoval y Rojas, primer Duque de Lerma.

Fue el más famoso de los Condes de Lemos. Antes de los 30 años y vuelto a España de las guerras de Flandes, fue nombrado durante el reinado de Felipe III, Presidente del Consejo de Indias el 7 de abril de 1603. En 1610 asumió el Virreinato de Nápoles, "en que dejara su padre tan buena memoria, y que él desempeñó con el mismo acierto hasta 1616".

Amante de las letras, fue el más brillante mecenas de su época; tuvo como secretario al célebre Lope de Vega, que también lo fue de su padre. Protector de Cervantes y amigo de Quevedo, Góngora, los hermanos Argensola, Mira de Amescua y Vicente Espinel, se rodé de darillanterséquito de literatos cuando marchó a Nápoles, nombrando como Secretario de Estado y Guerra del Virreinato a Lupercio Leonardo Argensola.

Con tan distinguida corte, a la que se sumaron escritores y aristócratas napolitanos, fundó con el erudito Juan Bautista Manso, Marqués de Villa, la renombrada Academia deglo Oziosi. En esta academia, el Conde de Lemos tuvo intensa actividad literaria, leyendo sus composiciones poéticas y alguna comedia. "Efectivamente, se sabe que escribió una obra teatral "La casa confusa" como también las redondillas que preceden al poema de Lope de Vega sobre San Isidro y asimismo una sátira en prosa titulada "El buho gallego". También se dice que fue autor de una descripción del gobierno de Quixos y Macas, en el Perú, en la época que ocupó la presidencia del Consejo de Indias". (2)

El Conde de Lemos regresó de Nápoles en 1616 para ocu-

(1) Francisco Fernández de Bethencourt. Historia Genealógica y Heráldica de la Monarqua

Española, Madrid, 1902, T.IV., pág. 551.

(2) Jorge Basadre. El Conde de Lemos y su tiempo, Lima, 1945, pág. 27. 
par la presidencia del Consejo Supremo de Italia hasta 1618 en que cesó en sus funciones a la caida politica de su suegro el Duque de Lerma. Apartado de la Corte se reliró a su palacio señorial de Monforte, donde pasó tres años. Falleció en Madrid el 19 de octubre de 1622, a la edad de 46 años.

Muerto el Conde, Lope de Vega hizo su elogio en su Laurel de Apolo, llamándolo "Claro y siempre amado, señor mio". En vida le había dirigido los siguientes versos donde reconoce sus méritos literarios:
Estilo superior, divina mano,
Pluma sutil de peregrino corte.
Arte divino, contrapunto en llano
Sois del mar de escribir lucido Norte,
Pero diréis que son lisonjas éstas.
Como me dan los aires de la Corte.
Aunque si son verdades manifiestas.
Diganlo las epistolas divinas
Que os escuché gon tal primor compuestas.

Estrecha y permanenfe anmstad unió al Conde de Lemos con los hermanos Argensola especialmente, con Lupercio Leonardo del que ya dijimosfuo su secretario en Nápoles. Compartieron la amistad entre los quehaceres políticos y literarios, estos últimos en las Bifflinzedasle weachemias donde participaron activamente. "Jorge Puccinelli Converso"

"La institución académica tiene en su origen platónico una indole que se deforma después" (1). Las nuevas academias se crearon en Italia en el siglo XIII hasta alcanzar su mayor auge a lo largo del XVI. Imitándolas, se establecieron en España a fines del XVI y principios del XVII constituyendo centros de irradiación cultural. "Es el período del Renacimiento y las academias sirven coino el vehículo eficacísimo para el cultivo y progreso de las artcs, las letras y las ciencias" (2).

Intensa actividad desplegó el Conde de Lemos en algunas academias tanto napolitanas como españolas, ya fomentándolas, ya actuando con sus propias composiciones o sirviendo de juez en los certámenes literarios.

En la academia denominada "Los Anhelantes", Lupercio Argensola, el íntimo amigo y colaborador del Conde, dictó al-

(1) José Gabriel. Historia de la Gramática, (Anexo a la revista "San Marcos"), Lima. 1949, pág. 83.

(2) José Sánchez. Academias literarias del Siglo de Oro español, Gredos, Madrid, pág. 12. 
gunas conferencias en las que hallamos algunos datos que nos demuestran el interés lexicológico del disertante, interés que también había de compartir don Pedro Fernández de Castro, y que lo indujo a componer el brevísimo Diccionario, ya mencionado, al describir una lejana y exótica provincia americana; descripción, que si bien es cierto tuvo una finalidad administrativa, no por eso cleja de tener relativo valor literario, aparte de la singular importancia que le encontramos como sugestivo documento para la lexicografía americana.

Argensola, en una de esas conferencias, al ocuparse de la historia, decía a sus contertulios, que era necesario imitar a Lipsio, el célebre filólogo y literato belga de la época, y que, así como describió la milicia, enseñó la forma de los ejércitos romanos, sus armas ofensivas y defensivas; qué era gálea, loriga, pilo, parma y las demás; qué soldados eran los vélites y los céleres; así, él necesitaba saber cómo eran las armas y las máquinas de los españoles y cómo usaban los paveses y las lanzas; qué cosa era pespunte y loriga, cómo formaban las mantas o gatas; qué máquinas era el funebol, el magaret, el trabuco y otros semejantes... Lucgo añadia: "No es cosa vergonzosa, señores, que habiéndoles ganado a vuesas mercedes sus mayores la nobleza, estado y hacienda que poseen, con esta milicia, armas e instrumentos ignoran to que son?... Vuelvo a decir que sería muy loable trabajjbotedca ảlgumo de vuesas mercedes... nos descubriese cómo éeanncceala cosa de éstas" (1).

Volviendo al Conde de Lemos, en 1603, a la edad de 27 años, fue nombrado Presidente del Consejo de Indias, alto cargo que desempeñó hasta 1609. Su cultura formada en el ambiente cortesano al lado de Lope de Vega, y de los más preclaros talentos de la época, le hizo apreciar que su recargada labor administrativa en el Consejo no estaba reñida con las actividades científico-literarias, sino que, por el contrario, se complenentaban vivificándose; y sacando partido de la lectura atenta de las comunicaciones llegadas de América, las vació en los moldes de su estilo literario. En efecto, en 1608, publicó una interesante "Descripción de la provincia de los Quixos", opúsculo sobre el cual el historiadur Jiménez de la Espada dice haberlo conocido a través de la siguiente nota de J. Bautista Muñoz: "En la librería de la catedral de Palencia había en 26 de jumio de $178 \mathbf{i}$ una relación impresa de la Provincia de los Quixos escrita por el Conde de Lemos y de Andrade, quien la dedica a su padre

(1) José Sánchez, Obra citada, pág. 24. 
con fecha de Madrid 16 de febrero de 1608. Es un tomito de 16 hojas, en 4? mayor, sin frontis ni lugar de impresión, aunque sin duda en Madrid. Va al principio un mapa grabado de dicha provincia" (Col. Muñoz, t. 93, f․ 156)(1).

Conocemos la curiosa publicación del Conde de Lemos por haberla transcrito Jiménez de la Espada en el primer tomo de sus "Relaciones Geográficas de Indias", referente al Perú, que hemos citado con frecuencia. Dicho artículo contienc una dedicatoria, un cortísimo vocabulario y la descripción acompañada de un mapa de la región de los Quixos.

En la dedicatoria, después de algunas consideraciones históricas, políticas y administrativas, manifiesta el autor que habiendo recibido el año anterior informaciones de América tomaba a su cargo darles forma y estilo convenientes, trabajo digno de su persona por el ministerio que ejercitaba; y, que en lugar de extenderse en cosas que puedan admirar y divertir, sólo le había vencido el gusto y la curiosidad en las notables y famosas de aquella provincia (de los Quixos), a fin de que la relación escrita, en caso de ser aprobada, pudiera servir de modelo a las otras descripciones. A continuaciø̆ir agrega:

"Algunas cosas convenientes á la Descripción perfecta de la Gobernación de los Quixos se han omitị̂o en esta relación, porque las informaciones de Indias vinieron Blgbiodènidetastranyo defecto es común im. perfección de todos los principiess,ugenflliçpeluerse en las segundas relaciones que se piclen y ahora PARA MAYOR INTELIGENCIA ME HA PARECIDO PONER AQUI UN DICCIONARIO DE LOS VOCABLOS PAR. TICULARES DE LAS INDIAS, Y POCO FAMILIARES EN ESPAÑA.

ARCABUCOS.- Montes de árboles espesos, y enetrados (sic).

BAHAREQUES.- Tabique de paja y barro.

CAMAYO.- Indio que vive en pueblo de españoles y en su lengua quiere decir forastero.

LA CORDILLERA.- Así se llaman las sierras que corren por el Perú desde Caracas hasta los términos de Chile.

DOTRINERO.- Cura que administra los Sacramentos a los Indios.

DOTRINA.- Beneficio de Indios.

ENCOMENDERO.- Persona a quien pagan los Indios el tributo.

(1) Jiménez de la Espada, Obra citada, pág. XCVIr. 
ENCOMIENDA. - Señorío de Indios tributarios.

ESCUPILES.- Armadura de algodón basteado, como corazas.

GUANDOS.- Son unas andas a manera de literillas descubiertas.

LOS MACAS.-Indios de una provincia que tienen este nombre, como en España los Castellanos, o los Andaluces.

RESERVADO.- Indio que por su vejez no paga tributo.

PRESERVADO O TRIBUTERO.- Indio que aun no tributa, por ser de poca edad.

REPARTIMIENTO.- Lo mismo que encomienda.

TRIBUTO.-La imposición que pagan los Indios por el vasallaje.

PARCIALIDAD.- Barrio.

INGA- El Rey del Perú.

LIMA.- Metrópoli de todo el Perú donde residen el Virrey, y el Arzobispo" (1).

A continuación del "Diccionario" trae la Descripción de la Provincia de los Quixos, dividida en cinco partes dedicadas a informar sobre lo moral, lo natural, lo eclesiástico, lo militar y sobre las ciudades de Bảézai, Avila, Artchidona y Sevilla del Oro.

Esta prolija e interesante descripción proporciona abundantes notas sobre historia, geografía, gobierno, economía, administración civil $\mathrm{y}$ eclesiástica, así como otras referentes a la producción, industria, etc. Trae también una curiosa descripción de las granadillas al referirse a las especies vegetales de la región.

La "Descripción de la provincia de los Quixos, ordenada y metódica, como hecha para que sirviera de modelo a las posteriores, aparte de los vocablos, materia del Diccionario, consigna otras voces americanas más que, a nuestro parecer, no fueron incluídas en el vocabulario, porque eran muy conocidas a través de anteriores relaciones y descripciones. En próximos artículos las mencionaremos entre los americanismos que figuran en las "Relaciones Geográficas" publicadas.

De todas maneras, los dieciocho vocablos particulares de las Indias y poco familiares en España, que el Conde de Lemos presentó en no muy estricto orden alfabético con el nombre de

(1) Jiménez de la Espada, Obra citada, pág. XCIX y C. 
"Diccionario", es una interesante muestra de la modalidad que fue adquiriendo el castellano en el Perú en cuanto al léxico, modalidad, que no tomando en cuenta los particularismos exclusivamente peruanos, obedece a una realidad generalizada en cada región de América donde se impuso la lengua del colonizador.

Nadie ignora que el español en América, mientras más se extendía, asimilaba mayor número de indigenismos ante la necesidad de denominar usos, costumbres, instituciones, animales y plantas del Nuevo Mundo. Asimismo, por el parecido o ciertas analogías, se fue dando nombres conocidos a las cosas nuevas ante el conflicto que se suscitaba entre los vicjos hábitos españoles y las nuevas experiencias. Por último, el español siguió y continúa derivando y formando nuevos términos en América, de acuerdo a sus propios y habituales procedimientos, de la misma manera como se fueron y van formando dentro de la propia Península, en un proceso ciertamente renovador o evolutivo.

Don Pedro Paz Soldán y Unanue, con el seudónimo de Juan de Arona, publicó en Londręs, en 1861, "Galería de novedades filológicas, Vocabulario de Pertanismos". En 1867 editó en Lima "Cuadros y episodios peruanos" en donde incluyó un "Indice alfabético de los términos peruanos en esta obra". Allí dice: "Entiendo por término peruano o peruanismo, no sólo aquellas palabras que realmente lo son, por ser derivadas del Quichua, o corrompidas del espanoptefanventazas por los criollos con el auxilio de la lengua castelfana, sino" también aquellas que, aunque muy castizas aluden a objetos o costumbres tan generales entre nosotros y tan poco comunes en España, que nos las podemos apropiar y llamarlas "peruanismos", como si no estuvieran en el Diccionario de la Academia Española" (1). Posteriormente, en 1862, en su "Bibliografía de Americanismos" (2) amplió el concepto que tenía acerca de estos vocablos: "Tambien considero peruanismos los nombres indígenas topográficos y de personas". Al año siguiente publicó su obra más completa con el título de "Diccionario de Peruanismos".

Con las anteriores publicaciones, Paz Soldán y Unanue fue el primer escritor que distinguió con mayor propiedad esta clase de términos usados en América y que hasta entonces se les llamaba "provincialismos". Así, pues, la palabra "Peruanismos" inicia la serie de "ismos" que identifican los particularismos geográficos según el país o región donde se les use.

(1) Juan de Arona (P.P.S. y U.) "Cuadros y episodios peruanos y otras poesías nacio-

les y diversas". Lima, 1867.

(2) Juan de Arona, Diccionario de peruanismos, Biblioteca de Cultura peruana, No 10. Desclée, De Brouwer, París, 1938. 
Sin discutir el acierto de Juan de Arona, al señalar las fuentes de donde provienen los peruanismos, y por extensión. los americanismos, en general, conviene a nuestro propósito destâcar las cuatro fuentes señaladas por él: $1^{?}$, los indigenismos, $2^{2}$, las voces inventadas por los criollos con el auxilio de la lengua castellana; 3 , las castizas que aluden a objetos o costumbres tan generales entre nosotros y tan poco comunes en España; y, 4?, los nombres topográficos y de personas.

Así, según esas cuatro fuentes, el "Diccionario" o vocabulario de don Pedro Fernández de Castro, Conde de Lemos, se agruparía en la siguiente forma:

1:- - bahareques. camayo, guandos, inga.

2:-_escupiles.

39--arcabucos, cordillera, dotrinero, dotrina, encomendero, encumienda, reservado. preservado, repartimiento, tributo, parcialidad.

4 ? - macas, Lima.

Al presentar el "Dicciohario" del Conde de Lemos, como la primera muestra sobre lexicografía americana en general, y peruana, en particular, en relación con el español, no ignoramos el valor linguiístico y filológico que siempre se ha reconocido a la documentación y Böbliat al contrario, tratamos de Prelievarveris" importancia dando una muestra de las posibilidades para proseguir la investigación acerca de la realidad lingüística americana. Pedro Benvenutto Murrieta, autor de "El Lenguaje peruano", anota: "Desde luego, en los cronistas y en los documentos de la conquista, hallamos con relativa frecuencia las voces indígenas que más o menos alteradas empezaron a usar los conquistadores. Posteriormente, en los escritos coloniales, son corrientes también, los peruanismos. Hasta listas de ellas con sus correspondientes definiciones coronan ciertas obras como las del famosísimo extirpador de idolatrías, Padre José de Arriaga, pero no existe ningún libro dedicado especialmente a ellos: no se les hace objeto de un estudio" (1).

Por nuestra parte, revisando cronistas, historiadores y variada documentación, dimos con el "Diccionario" del Conde de Lemos, hecho que no constituye un hallazgo ni una primicia porque es muy conocida la obra de Jiménez de la Espada, pero por otro lado, no conocemos ningún artículo ni comentario acerca

(1) Pedro Benvenutto Murrieta, El Lenguaje Peruano, Lima, 1946, pág. 7. 
del valor lingüístico que encerraban las "Relaciones Geográficas de Indias", al incluirse en ellas, nada menos, que un vocabulario llamado "Diccionario" por su autor, y que viene a constituir el más lejano aporte a la lexicografia americana.

Puesta nuestra atención en el "Diccionario" del Conde de Lemos, hemos revisado los diccionarios de Americanismos de Malaret y de Santa María, la obra de Toro y Gisbert, gran número de diccionarios y vocabularios de voces americanas, el "Tesoro Lexicográfico" de Gili Gaya, el Diccionario etimológico de Corominas, y en ninguna de sus bibliografías se menciona el trabajo de 1608. Por último, hemos consultado la "Bibliografía de la Linguística Española" de Homero Serís, la más completa obra sobre la materia, y nada menciona al respecto. Esta última y especial circunstancia es la que nos ha movido a escribir el presente artículo, con el que nos proponcmos rescatar el asunto y el mérito del Conde de Lemos, efectivos valores para la linguística española y el común destino cultural hispanoamericano.

Homero Seris, al enumerar en su obra la bibliografia sobre "Americanismos" señala que Ferpández de Oviedo (De la natural historia de las Indias, Toledo 1526; y la Historia General de Indias, 1535-1557) "utiliźó muchas palabras del lenguaje usado en América, pero no hizo un Vocabulario. De hacerlo hubiera sido el primero de americanismos" (4). Sin embargo, en 1608, el entonces Presidentecafte Corisejo Supremo de Indias, don Pedro Fernández de Castro, Conde de Lemos, fue el primero que compuso un "Diccionario" de peruanismos, que virtualmente fue también, el primerc de Americanismos.

(1) Homero Serís. Bibliografía de la Lingüistica Española, Bogotá, 1964, pág. 698. 